\title{
Thermal study of injection moulded ring
}

\author{
GAYATRI KANSAL, S K ATREYA and P N RAO* \\ Instrument Design Development Centre, Indian Institute of Technology, Hauz \\ Khas New Delhi 110016, India \\ * School of Mechanical Engineering, MARA Institute of Technology, 40450 \\ Shah Alam, Malaysia \\ e-mail: vistelcom@bol.net.in; gayatrikansal@hotmail.com
}

MS received 9 April 1997; revised 2 June 1998

\begin{abstract}
Application of CAD/CAM techniques to injection mould design is feasible and required now in view of the low cost of computers and the need to develop moulds in the shortest possible time. For this purpose a simpler thermal analysis of an injection mould is desirable, as most of the mould makers in Asia are in the category of small manufacturing units. This paper presents a thermal model of injection moulds for axisymmetric components using polar co-ordinates. This model could be utilised for studying the shrinkages, thermal residual stresses and cooling channels. A design example is presented to explain the method of application of the developed model.
\end{abstract}

Keywords. Injection moulded ring; thermal residual stresses; thermoplastics.

\section{Introduction}

For successful fabrication of plastic parts by the injection moulding process, optimum cooling and solidification must be accomplished in the shortest possible time. Injection moulding is one of the most efficient techniques for making thermoplastics. In this technique, molten polymer is forced inside the shaped cavity and after solidification and cooling the component is ejected. If a plastic part is cooled in too short a period of time, it may deform or flow upon release from the mould cavity. On the other hand, if the part is cooled for an excessive period of time, production costs become prohibitive and warpage may occur. Hence a cooling model is essential for optimizing the cooling rate, and is also necessary for automation of the injection moulding process. The thermal history of the melt, particularly the melt temperature, temperature of the mould and cooling rate, determines the microstructure and mechanical properties of injection-moulded plastic parts.

Siegmann et al (1981) studied the effect of the thermal history on the properties of moulded components and found that they are affected by the total temperature difference during cooling. Coxon \& White (1980) studied semicrystalline polymers, i.e., 
polypropylene, and found that the residual stresses depend on the temperature difference between the melt temperature and the mould temperature.

Rigdahl (1976) presented a finite element method for the study of polystyrene rectangular thin plate. Kamal \& Lafleur (1982) developed a cooling model that incorporates the effect of crystallinity kinetics during all stages of solidification while moulding. Latent heat of solidification was calculated from the specific heat within the cooling process, which was a function of the derivative of crystallinity with respect to temperature. Specific heat of the melt and the solid were assumed to be independent of the cooling process. Study of the cycle time in injection moulding of filled thermoplastics was carried out by Boldizar \& Kubat (1986). Measurement of cooling time was based on the assessment of recovery of the ejector marks for samples ejected after varying times of cooling.

Injection moulding with the molten polymer solidifying in the cavity is a major problem for which straight forward analytical solutions can only be obtained for very simple cases. Ockendon \& Hodgkins (1975) described the unsteady-state transfer of heat under pressure in a melt, which exhibits variable properties. Since heat transfer is accompanied by phase transformation, the resulting nonlinear partial differential equations, which describe the system are not amenable to explicit analytical solutions. Hence numerical methods are employed for arriving at the solution.

The principal mathematical problem encountered during solidification of the polymer in the cavity is the presence of a solid-liquid interface within the polymer and the need to calculate its position throughout the cooling cycle. Extensive amount of work is available on solving the heat transfer problems associated with phase change.

The concept of enthalpy is defined as sum of the sensible heat and the latent heat for a phase change. It has frequently been used in numerical modelling of phase-change problems. An important advantage of introducing enthalpy is that the phase condition can be implicity taken into account. As a result, numerical solutions of the problem can be achieved in an easier way, since it is no longer necessary to track the moving phase boundary. In the present paper, to carry out the temperature study in the cavity, finite difference technique is discussed. A model has been simulated ignoring the injection stage. The phase-change problem has been solved using the enthalpy method.

\section{Formulation of the problem}

In any moulding process, temperature is the basis of most of the studies which are to be carried out, such as thermal stresses, cycle time study etc. In the present paper, a cylindrical co-ordinate system in three dimensions is used to calculate the temperature inside the mould cavity. The present system has been developed using the finite difference method. Some of the advantages and disadvantages of these methods are as below. (Shamsunder \& Sparrow 1975).

Advantages of finite difference method (FDM): (1) It is easy to understand and apply.

(2) Computer memory requirements are small.

Disadvantages: Irregular and complicated shapes are difficult to handle.

Advantages of the finite element method (FEM): (1) The flexibility of application is the 
most favourable point of FEM. There is no need to have uniform mesh size. Irregular shapes are no more difficult to treat than regular shapes.

Disadvantages of FEM: (1) The development of any finite element computer code involves lot more preparatory work than that for corresponding finite difference work.

(2) It requires considerable memory for storage of coefficient matrices.

(3) The finite element method is more sensitive to numerical round off than finite difference method, and all calculations are to be made in double precision.

Therefore it was decided to use FDM for developing a PC-based model.

During the solidification of the polymer, solid-liquid interface is encountered. Of the number of solution methods available for the phase change problem, the enthalpy method was found to be the most suitable. The present paper is organised as follows.

(1) Mathematical formulation with general equations.

(2) A case study of a ring with an inside hole.

The results of the above case developed using FDM are verified by modelling them on the commercially available finite element package called P3/PATRAN. Due to the nonavailability of the thermal analysis module in P3/PATRAN in our installation, thermal analysis was not done. However the stress pattern can be verified using the temperature distribution results of the developed package on the PATRAN advanced-FEA module.

\subsection{Assumptions}

(1) At zero time, temperature at all the points inside the cavity was assumed to be equal to the melt temperature (Kenig \& Kamal 1970).

(2) Only half of the component is analysed, where the product is symmetric with respect to the $\mathrm{X}$-axis.

(3) The heat transfer increases, when liquid plastic is injected under pressure, due to convection. To take care of this effect, an effective thermal conductivity of the melt has been defined by Mizikar (1967), Sharir et al (1980), and Westby (1980). The value of effective thermal conductivity used by Westby (1980) is 0.25 in C.G.S. units, while, Mizikar used a value of seven times the thermal conductivity of the liquid. Sharir et al (1980) used a value five times the thermal conductivity of liquid alloy. In the present case the experimental and the published results matched when the conductivity was taken to be five times that of the liquid.

\section{Mathematical formulations}

\subsection{Thermal analysis}

The general three-dimensional equation for heat conduction for cylindrical co-ordinate system in a solid body is given by

$$
\frac{1 \partial}{r \partial r}\left(r K \frac{\partial \phi}{\partial r}\right)+\frac{1 \partial}{r^{2} \partial \theta}\left(K \frac{\partial \phi}{\partial \theta}\right)+\frac{\partial}{\partial Z}\left(\frac{\partial \phi}{\partial Z}\right)+Q=\rho c_{p} \frac{\partial T}{\partial t},
$$


where $\phi=$ temperature in ${ }^{\circ} \mathrm{C}, r=$ radius, $C_{p}=$ specific heat $\left(\mathrm{cal} / \mathrm{g}^{\circ} \mathrm{C}\right), \rho=$ density $\left(\mathrm{g} / \mathrm{cm}^{3}\right), t=$ time $(\mathrm{s})$.

In order to eliminate the above nonlinear equations and keep track of the moving boundary, two variables have been defined.

$$
\begin{aligned}
& T=\int_{0}^{\Phi}\left(K / K_{0}\right) \partial \Phi, \\
& H=\int_{0}^{\Phi} C_{P} \partial \Phi
\end{aligned}
$$

where $T=$ modified temperatire, $H=$ heat content or enthalpy $(\mathrm{cal} / \mathrm{g}), K_{0}=$ thermal conductivity of solid at $0^{\circ}$.

The substitution in the form they are applied are

$$
\begin{aligned}
K & =K_{0}(\mathrm{~d} T / \mathrm{d} \Phi), \\
C_{P} & =\mathrm{d} H / \mathrm{d} \Phi .
\end{aligned}
$$

The concepts of modified temperature and enthalpy were used by Sarjant \& Slack (1954) and Westby (1980).

By substituting (4) and (5) in (1), the final equation becomes

$$
\frac{K_{0}}{\rho}\left(\frac{1 \partial}{r \partial r}\left(r \frac{\partial T}{\partial r}\right)+\frac{1 \partial}{r^{2} \partial \theta}\left(\frac{\partial T}{\partial \theta}\right)+\frac{\partial}{\partial Z}\left(\frac{\partial T}{\partial Z}\right)\right)+\frac{Q}{\rho}=\frac{\partial H}{\partial t} .
$$

The central difference technique and the mesh shown in figure 1 are used to modify (6) to the form given below

$$
\begin{aligned}
H^{t+1}(I, J, L)= & H^{t}(I, J, L) \\
+ & \frac{k \Delta t}{\rho}\left[\frac{\left(T^{t}(i+1, j, L)-2 T^{t}(i, j, L)+T^{t}(i-1, j, L)\right)}{r^{2} \mathrm{~d} \theta^{2}}\right. \\
& \times \frac{\left(T^{t}(i, j+1, L)-T^{t}(i, j-1, L)\right)}{2 r \mathrm{~d} r} \\
+ & \frac{\left(T^{t}(i, j+1, L)-2 T^{t}(i, j, L)+T^{t}(i, j-1, L)\right)}{\mathrm{d} r^{2}} \\
& \left.+\frac{\left(T^{t}(i, j, L+1)-2 T^{t}(i, j, L)+T^{t}(i, j, L-1)\right)}{\mathrm{d} z^{2}}\right],
\end{aligned}
$$

where $\mathrm{d} r=$ increment in the radial direction, $\mathrm{d} \theta=$ increment in the angular direction, $\mathrm{d} z=$ increment along the height, $r=$ radius from the centre, $K=$ thermal conductivity, $\Delta t=$ time increment, $\rho=$ density.

Equation (7) is solved using the explicit method. The condition for stability is

$$
\Delta t \leq 1 /\left[\frac{2 K_{0}}{\rho} \frac{\partial T}{\partial H}\left(\frac{1}{\Delta x^{2}}+\frac{1}{\Delta y^{2}}\right)\right],
$$

Boundary conditions used are as follows,

$$
\partial T / \partial n=-(h / K)\left(T_{B}-t_{A}\right) .
$$




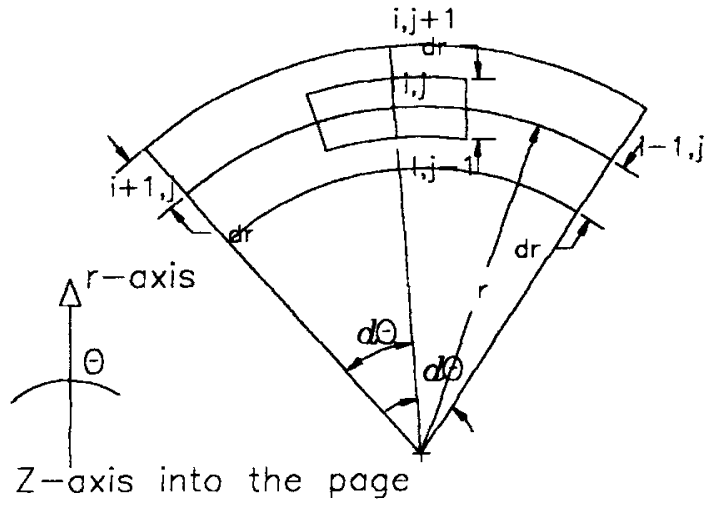

Figure 1. Cylindrical coordinates.

\subsection{Cooling channels}

To study the effect of cooling on the temperature distribution, it is important to calculate convective heat transfer co-efficient, $h$, with the procedure given below.

Cooling channels are assumed to be tubes. The Reynold number is given by

$$
\operatorname{Re}_{d}=\rho \mu_{m} d / \mu
$$

where $\rho=$ density, $\mathrm{g} / \mathrm{cm}^{3}, m=$ dynamic viscosity, $\mathrm{g} / \mathrm{cm} . \mathrm{s}, m_{m}=$ mean velocity, $\mathrm{cm} / \mathrm{s}$, $d=$ diameter of the cooling channel, $\mathrm{cm}$.

Diameter of the cooling channels is decided by the rules based on the practical experience of tool-room designers. These rules are given in figure 2. The diameter of the cooling

(a)

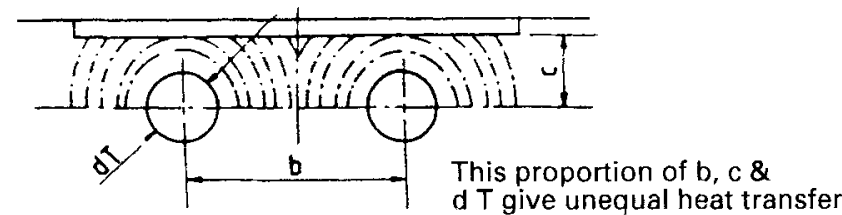

(b)

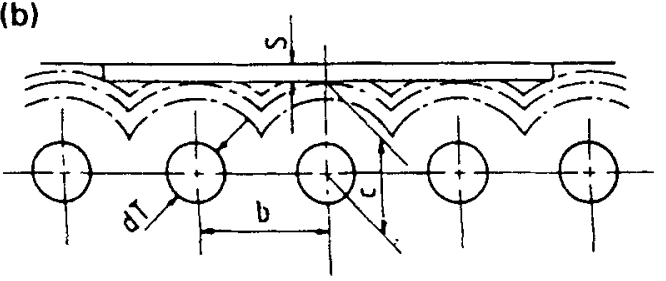

WALL THICKNESS

CHANNEL DIA (dT)

$\begin{array}{lr}2 \mathrm{~mm} & 8-10 \mathrm{~mm} \\ 4 \mathrm{~mm} & 10-12 \mathrm{~mm} \\ 6 \mathrm{~mm} & 12-15 \mathrm{~mm} \\ \text { distance } \mathrm{C}=2-3 \times \mathrm{dT} & \\ \text { distance } \mathrm{b}=\text { max. } 3 \times \mathrm{dT} & \end{array}$

Figure 2. Relation between diameter and distance of cooling channels (a) unequal heat transfer, (b) almost uniform heat transfer. 
channel varies according to the thickness of the cavity. The distance between the cooling channels is decided in such a way that the heat transfer is uniform, as the purpose of providing these channels is defeated if the heat transfer is unequal, as it will give rise to larger thermal stresses. The Nusselt number is calculated as follows.

$$
\begin{aligned}
\mathrm{N}_{d} & =0.023 \mathrm{Re}_{d}^{0.8} \operatorname{Pr}^{0.4}, \\
\operatorname{Pr} & =\text { Prandtl number }=5.85 .
\end{aligned}
$$

Convective co-efficient can be calculated as

$$
h=K \mathrm{~N}_{d} / d,
$$

where $K=$ thermal conductivity, $\mathrm{cal} / \mathrm{cm} \mathrm{K}$.

Value of $h$ is calculated at the steel mould and water interface. Flow is assumed to be laminar inside the cooling channel. At the cooling channel, mould interface boundary equations are given by

$$
\partial T / \partial n=-(h / k)\left(T_{B}-t_{A}\right) .
$$

\section{Case study}

A ring of diameter $100 \mathrm{~mm}$ and thickness $5 \mathrm{~mm}$ with a hole of diameter $60 \mathrm{~mm}$ in the centre as shown in figure 3 is chosen for the study. The ring is symmetrical about the $X-X$ and the $Y-Y$ axes. It is sufficient to analyse only half of the ring, since the analysis can be extended to the other half because of the symmetry.

The following conditions are used to eliminate the points lying outside the cavity as shown in figure 4 ,

$$
t(-2, j)=t(2, j) \quad \text { and } t(-n, j)=t(n, j),
$$

where $j$ is any point in the radial direction.

The cavity is divided into three layers along the thickness, i.e. the bottom, centre and top layer at a distance of $2.5 \mathrm{~mm}$. The ring is divided into nine sections along the radius at every $2.5 \mathrm{~mm}$ and into 13 sections in the angular direction, i.e., at an angle of $15^{\circ}$.

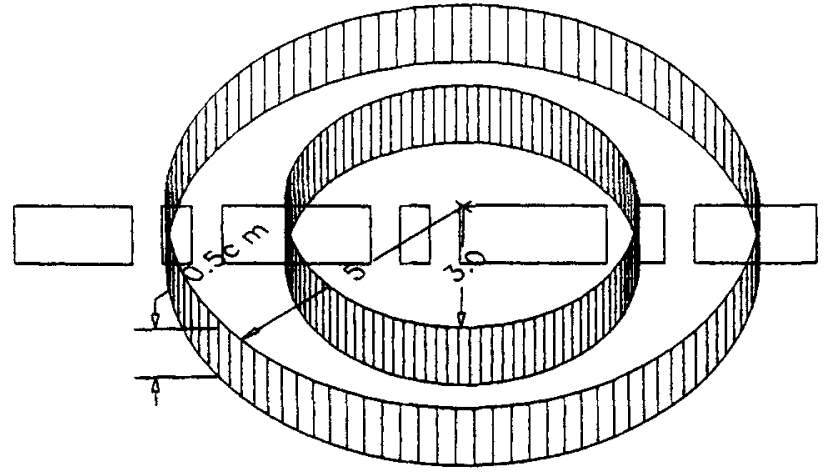

Figure 3. Ring for analysis (dimensions in $\mathrm{cm}$ ). 


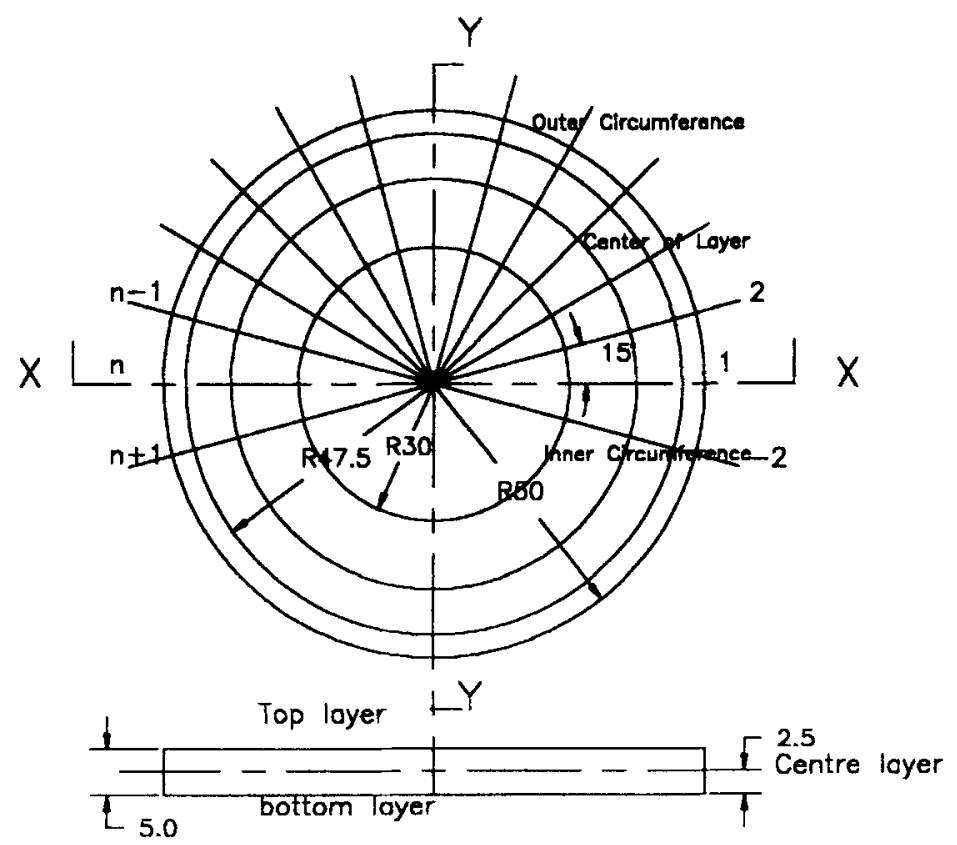

Figure 4. Segments of the ring (dimensions in $\mathrm{mm}$ ).

As explained above, the geometry of the ring is divided into different points. Points are stored by numbers as explained above. Temperature distribution, thermal stress analysis and effect of cooling channels are studied at these points. The observations are given in the following sections.

\subsection{Thermal analysis}

The results obtained from the computation are presented in tables 1 and 2 for different layers of the component. From these tables it can be observed that the centre layer cools slowly compared to the top and bottom layers. The top and bottom layers show the same temperature patterns, since both have the same contact with the metal. From the above study it is observed that the temperature for different angles but the same radius is the same for all the points. Tables 1 and 2 give temperatures for any one point at one particular

Table 1. Temperature in the top layer of the ring with respect to time without cooling channels

\begin{tabular}{ccc}
\hline Time & Point in the radial direction & Temperature \\
\hline 5 & 11 & 373.821 \\
10 & 11 & 343.823 \\
15 & 11 & 331.536 \\
20 & 11 & 319.78 \\
25 & 11 & 311.261 \\
30 & 11 & 306.686 \\
35 & 11 & 304.159 \\
\hline
\end{tabular}


Table 2. Temperature in the middle layer of the ring with respect to time without cooling channels.

\begin{tabular}{ccc}
\hline Time & Point in the radial direction & Temperature \\
\hline 5 & 11 & 460.083 \\
10 & 11 & 415.541 \\
15 & 11 & 381.561 \\
20 & 11 & 344.338 \\
25 & 11 & 323.152 \\
30 & 11 & 312.365 \\
35 & 11 & 307.012 \\
\hline
\end{tabular}

radius for the top and middle layer respectively. In these tables, temperatures shown are for an interval of five seconds. It is also observed that the outer circumference (i.e., the layer at $50 \mathrm{~mm}$ radius) is cooling faster as compared to the inner circumference (i.e., the layer at radius $30 \mathrm{~mm}$ ). This difference in cooling is the result of the difference in the area of the two circumferences.

Cooling is uniform in the angular direction because all the points at a given radius have the same contact with the metal. Points along the outer circumference of the ring are cooling faster than the points along the inner circumference because of the larger area of contact.

Figure 5 is showing the difference between the points at maximum and minimum temperatures with respect to time for the bottom and the middle layer. The maximum temperature difference observed is $51 \mathrm{~K}$ in the case of the bottom layer whereas in the centre layer it is $107 \mathrm{~K}$. This temperature difference gives rise to thermal stresses. Figure 5 makes it clear that the temperature difference between the hottest and coolest point of the cavity decreases as time increases in case of the bottom and middle layers. From the above results it can be interpreted that after a certain time when the boundaries are already cool,cooling progresses more rapidly towards the interior points of the cavity.

From the above analysis, temperature distribution at different points is known in all the three directions. Then this analysis is further extended to thermal stress analysis. The stress analysis is carried out as a two-dimensional and plane stress problem, therefore temperatures with respect to time are stored for different layers.
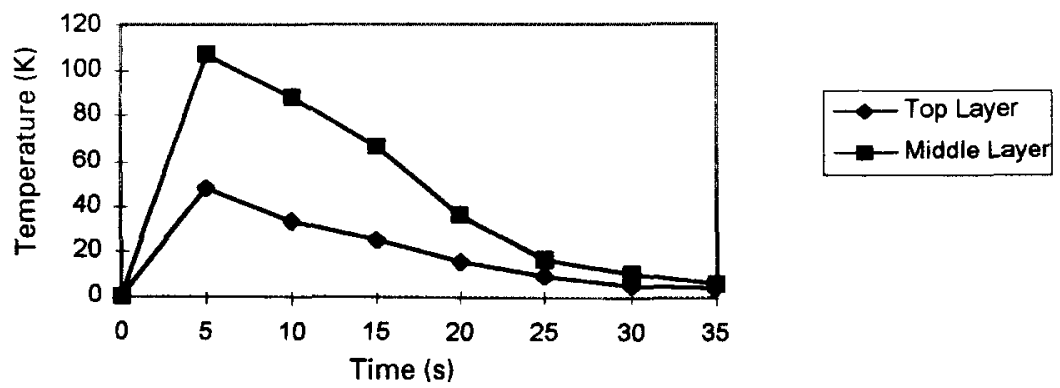

Figure 5. Temperature difference vs. time curve between the hottest and the coolest point of ring. 


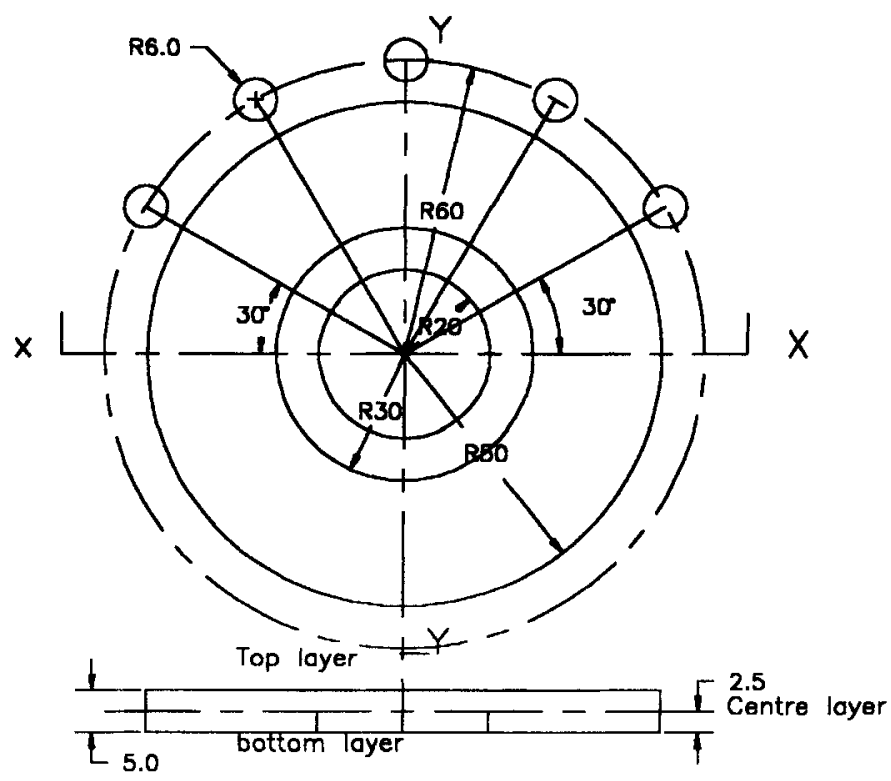

Figure 6. Position of cooling channels (dimensions in $\mathrm{mm}$ ).

\subsection{Effect of cooling channels}

In previous sections studies were carried out without cooling channels. The effect of cooling channels in two different orientations is studied in this section.

In the first case, two cooling channels of radii $6 \mathrm{~mm}$ are placed at angles of $60^{\circ}$ and $120^{\circ}$ respectively. The centres of these two channels are $60 \mathrm{~mm}$ from the centre of the cavity.

In the second case, six cooling channels are provided. One of them is positioned in the centre of the cavity inside the hole. The radius of the centre cooling channel is $20 \mathrm{~mm}$ and is positioned at a distance $2.5 \mathrm{~mm}$ below the top surface of the cavity. The other five cooling channels are placed along the periphery with their centres $60 \mathrm{~mm}$ from the centre of the cavity and at angles of $30^{\circ}, 60^{\circ}, 90^{\circ}, 120^{\circ}$ and $150^{\circ}$ respectively as shown in figure 6 . All the cooling channels placed along the periphery are of $6.0 \mathrm{~mm}$ radius and of the same height as the central cooling channel. Heat transfer coefficient, $h$, is calculated for the centre and the other peripheral cooling channels using (8) to (11).

In the present two cases, i.e. in the presence of cooling channels, all the points along a particular radius are not at the same temperature, as discussed in the previous section. This is because of the positioning of the cooling channels. However all the points have temperatures very close to each other along a given radius in the second case. In the first case, because the cooling is not as uniform as in the second case, temperature variation is more giving rise to higher stresses than in the second case. Tables 3 and 4 give the temperatures at different radii with respect to time for the middle and bottom layers for the second case. These tables give temperature for any one point, which has the highest temperature along the radius.

Figures 7 and 8 display the temperatures without and with cooling channels. From these figures it is clear that the cavity cools faster and more uniformly with the cooling channels 
Table 3. Temperature in the top layer of the ring with respect to time with cooling channels.

\begin{tabular}{lcc}
\hline Time & Point in the radial direction & Temperature \\
\hline 5 & 11 & 350.146 \\
10 & 11 & 333.209 \\
15 & 11 & 323.204 \\
20 & 11 & 313.303 \\
25 & 11 & 307.744 \\
30 & 11 & 304.747 \\
35 & 11 & 303.096 \\
\hline
\end{tabular}

and results in a shorter cycle time and lower residual thermal stresses. Comparison of the results for two different cases of cooling channels show that from the design point of view the second option of the cooling channels is much better compared to the first. This analysis also gives a method by which an estimate of the residual stresses in the components can be made. This would help in the designing of better moulds.

\section{Comparison of results}

Published results are not available for the above developed case in the literature. To verify the validity of the above, Finite Element Analysis was carried out using P3/PATRAN developed by PDA Engineering, USA. This package has different modules. For the present case, ADVANCED/FEA module is used. Due to the non-availability of the thermal module thermal stress patterns are developed to verify the model. For this, temperatures are loaded from the data obtained through finite difference thermal analysis model. Finite element mesh and the boundary conditions are shown in figure 9 . In the present case, quantitative matching is not carried out because different temperature dependent parameters cannot be given in tabular form. Hence qualitative study of the thermal stresses is performed based on the temperature obtained from the thermal analysis module (figures 10-12). Thermal patterns obtained from the thermal analysis module are utilised to calculate the stresses in the mould using both the theoretical as well as the PATRAN approach. It is seen that the stress pattern matches very well. This validates the above developed thermal model which can be used as a first approximation for the mould design purpose.

Table 4. Temperature in the middle layer of the ring with respect to time with cooling channels.

\begin{tabular}{lcc}
\hline Time & Point in the radial direction & Temperature \\
\hline 5 & 11 & 451.587 \\
10 & 11 & 404.418 \\
15 & 11 & 366.024 \\
20 & 11 & 332.108 \\
25 & 11 & 315.933 \\
30 & 11 & 308.328 \\
35 & 11 & 304.71 \\
\hline
\end{tabular}




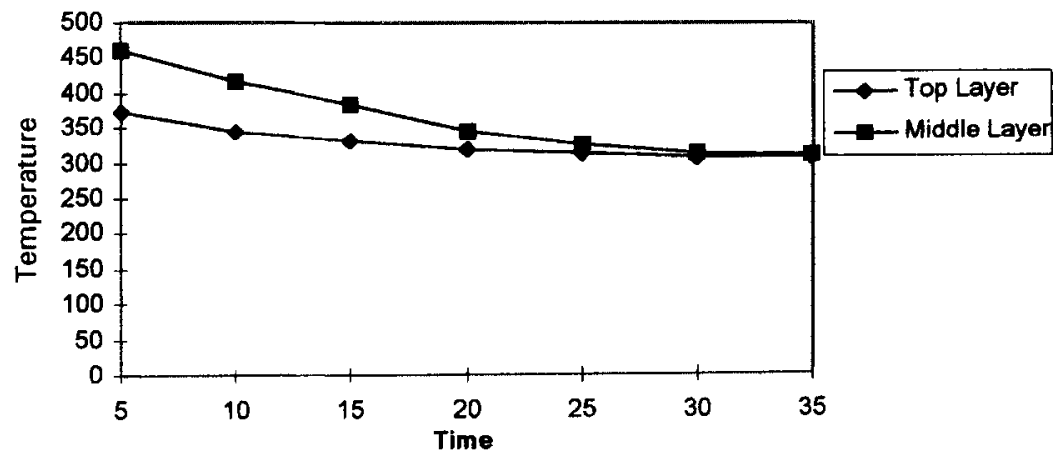

Figure 7. Time vs. temperature curve without cooling channel in the ring.

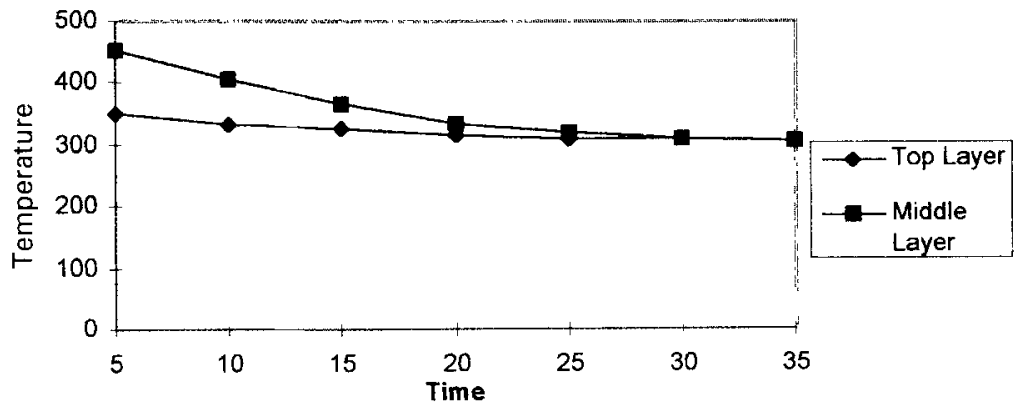

Figure 8. Time vs. temperature curve with cooling channels for the ring.

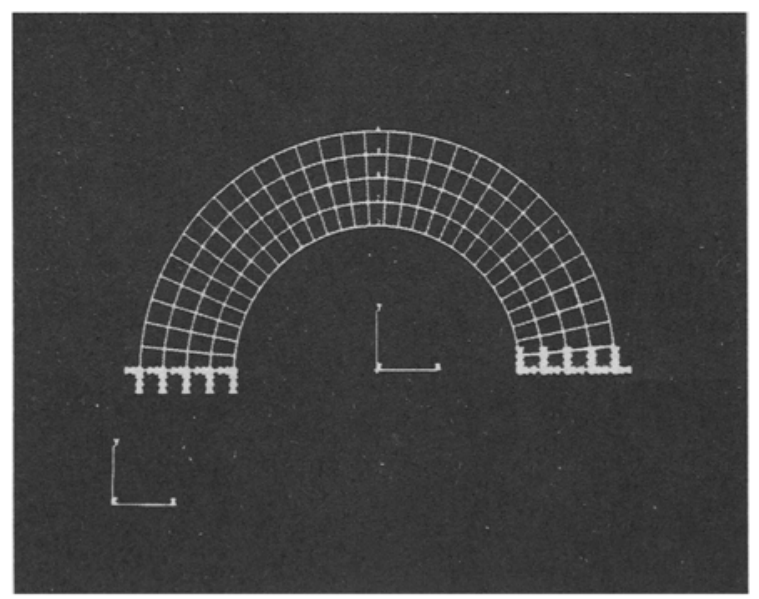

Figure 9. Finite element mesh. 


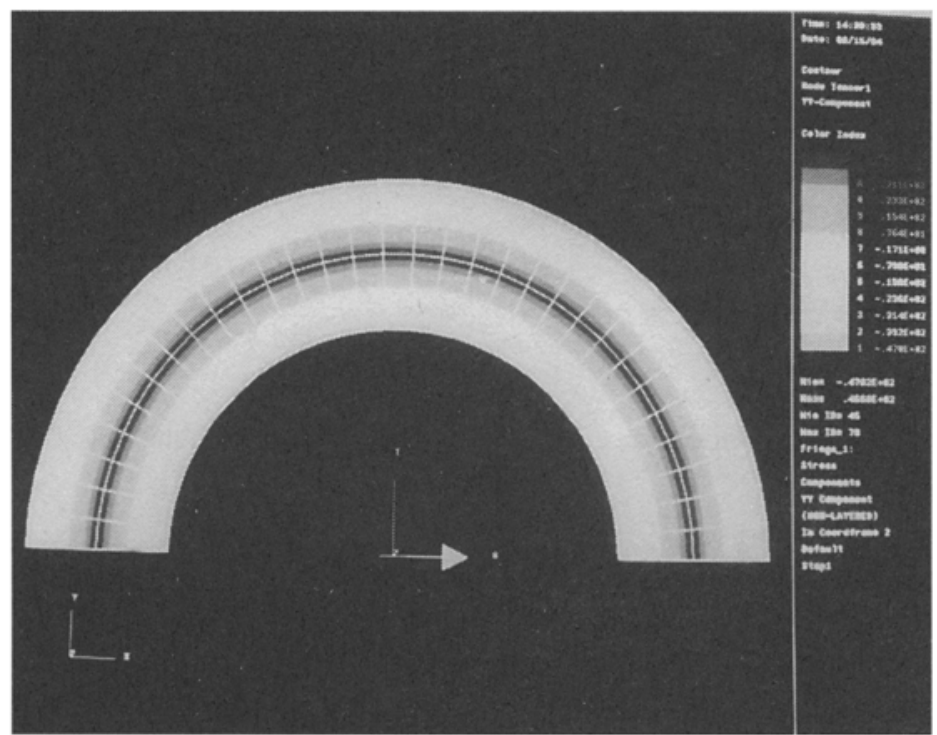

Figure 10. Hoop stress pattern.

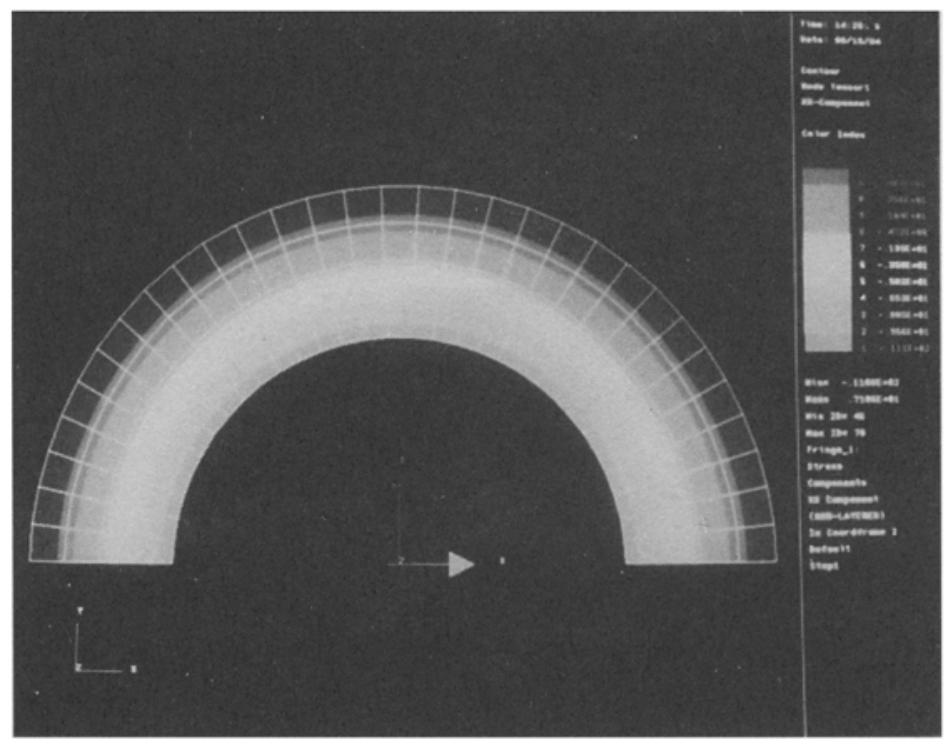

Figure 11. Radial stress pattern. 


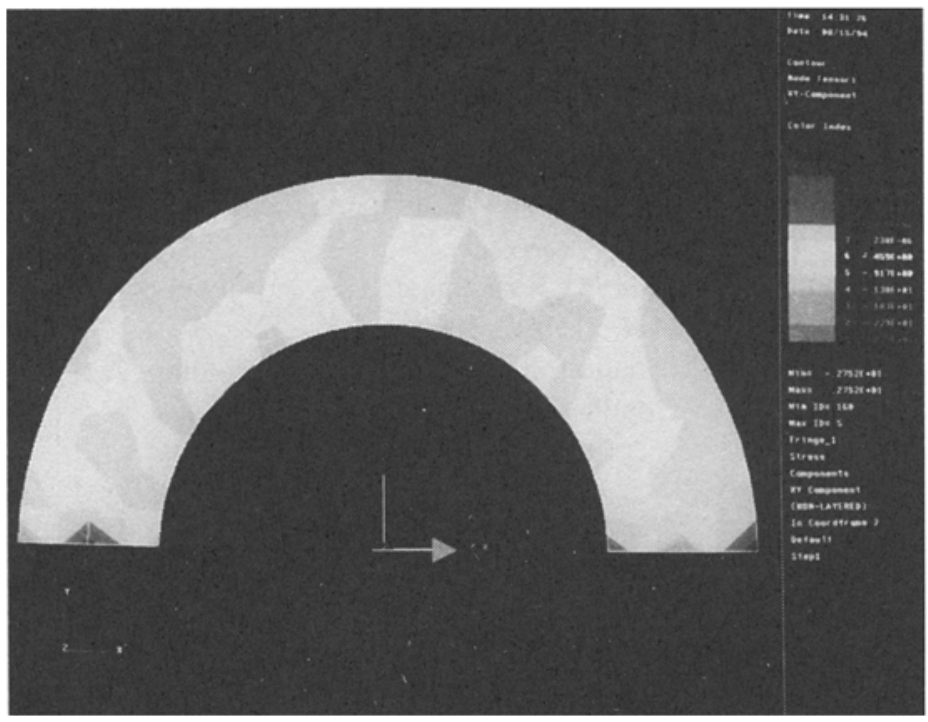

Figure 12. Shear stress distribution.

\section{Conclusion}

Literature review on the importance and development of CAD systems for injection moulds is carried out and it is observed that the majority of the systems reported are FEM-based and that the presence of the CAD system is mandatory. It is necessary to develop a simpler system for mould designing on a IBM PC-AT to be of use to a large spectrum of users.

A mathematical model based on finite difference method and enthalpy method using central difference technique has been developed to study an injection-moulded polystyrene ring. The effect of cooling channels has also been studied on the ring. It was seen that the proper placement of cooling channel could greatly reduce the temperature difference.

\section{References}

Boldizar A, Kubat J 1986 Measurements of cycle time in injection molding of filled thermoplastics. Polym. Eng. Sci. 26: 877-885

Coxon L D, White J R 1980 Residual stresses and aging in injection molded polypropylone. Polym. Eng. Sci. 20: 230-236

Kamal M R, Lafleur P G 1982 Computer simulation of injection molding. Polym. Eng. Sci. 22: 1066-1074

Kenig S, Kamal M R 1970 Cooling molded parts - A rigorous analysis. SPE J. 26: 50-57

Mizikar E A 1967 Mathematical heat transfer model for solidification of continuously cast steel slabs. Trans. Metal. Soc. AIME 239: 1747-1753

Ockendon J R, Hodgkins W R 1975 Moving boundary problems in heat flow and diffusion (Oxford: University Press)

Rigdahl M 1976 Calculation of residual thermal stresses in injection molded amorphous polymers by the finite element method. Int. J. Polym. Mater. 5: 43-57 
Sarjant R J, Slack M R 1954 Internal temperature distribution in the cooling and reheating of steel ingots. J. Iron Steel Inst. 177: 428-444

Shamsundar N, Sparrow E M 1975 Analysis of multidimensional conduction phase change via the enthalpy model. J. Heat Transfer. Trans ASME 97: 333-340

Sharir V, Grill A, Pelleg J 1980 Computation of temperatures in thin tantalum sheet welding. Metal. Trans. 118: 257-265

Siegmann A, Buchman A, Kenig S 1981 Residual stresses in polymers, II. Their effect on mechanical behaviour. Poly. Eng. Sci. 21: 997-1002

Westby O 1980 Temperature distribution in the workplace by welding. A report, Marine Technology Centre, University of Trondheim, Norway 